(O2019 IEEE. Personal use of this material is permitted. Permission from IEEE must be obtained for all other uses, in any current or future media, including reprinting/republishing this material for advertising or promotional purposes, creating new collective works, for resale or redistribution to servers or lists, or reuse of any copyrighted component of this work in other works. This is the author's version of an article that has been published in the conference proceedings. The final version of record is available at https://doi.org/10.1109/ICPHYS.2019.8780353 


\title{
RANSAC for Exchanging Maps in Multipath Assisted Positioning
}

\author{
Markus Ulmschneider and Christian Gentner \\ German Aerospace Center (DLR), Institute of Communications and Navigation \\ Muenchner Str. 20, 82334 Wessling, Germany \\ \{markus.ulmschneider,christian.gentner\}@dlr.de
}

\begin{abstract}
The classical approach to the multipath problem in positioning algorithms is to try to mitigate the influence of multipath components (MPCs) on the line-of-sight (LoS) path. Over the last years, a contrary approach has emerged with multipath assisted positioning, where MPCs of terrestrial signals are regarded as LoS signals from virtual transmitters. Thus, the spatial information in MPCs is exploited for localization. The locations of the physical and virtual transmitters can be estimated together with the user position using simultaneous localization and mapping (SLAM). To decrease the possibly long convergence time of SLAM, maps of transmitters can be exchanged among users. The information in a map can then be fused with the user observations. However, in the general case, the users do not know their starting locations and/or headings, and therefore are in their own local coordinate systems. When maps of transmitters are exchanged, the relative rotation and translation between the coordinate systems of a user and a received map need to be estimated. Within this paper, we propose a variant of the random sample consensus (RANSAC) algorithm for this estimation as it is very robust against outliers. We show by simulations in an indoor scenario that RANSAC increases the accuracy significantly.

Index Terms-Channel-SLAM, RANSAC, simultaneous localization and mapping
\end{abstract}

\section{INTRODUCTION}

The amount of services and applications that require a precise localization of a user has grown considerably over the past years. Global navigation satellite systems (GNSSs) can satisfy the needs of many such services in conditions with a clear view to the sky. Though, in GNSS denied scenarios such as in urban canyons or indoors, alternative methods for precise localization have to be found and developed. One approach is to use terrestrial radio signals for positioning. Such signals can be wireless local area network (WLAN) or cellular signals, for example [1]. In particular in indoor scenarios, these signals are subject to multipath propagation. They are reflected, scattered or diffracted by structures in the environment.

Typically, multipath propagation deteriorates the performance of positioning systems [2]. Over the last years, however, a new approach named multipath assisted positioning has emerged. While standard approaches in localization try to remove the influence of multipath components (MPCs) on the line-of-sight (LoS) path, the spatial information contained in MPCs is exploited in multipath assisted positioning. Each MPC can be regarded as a signal transmitted in a pure LoS condition by a virtual transmitter. The locations of the virtual transmitters are in general unknown. They may be calculated in advance if the location of the physical transmitter and the geometry of the environment are known [3], [4]. If no such information is available, the locations of both the physical and virtual transmitters can be estimated jointly with the user position using simultaneous localization and mapping (SLAM).

The authors of [5] have introduced such an approach named Channel-SLAM. Channel-SLAM does not differentiate between physical and virtual transmitters. Each signal component that is received in a multipath environment is regarded as being transmitted by a transmitter in a LoS condition. Accordingly, there is no differentiation between the LoS component and the MPCs.

In certain scenarios such as in shopping malls or museums, a high amount and fluctuation of users can be expected. Thus, maps of estimated physical and virtual radio transmitter locations may be shared. When a user receives a transmitter map, the a-priori information on transmitters can decrease the convergence time and increase the positioning performance of SLAM. If the starting location and direction of a user are unknown, the relation of the coordinate system of the user and the coordinate system of a received map is unknown as well. Hence, the rotation and translation parameters relating the two coordinate systems need to be estimated. We denote this estimation together with finding correspondences among transmitters in two maps by map matching.

In [6], we have presented a first approach to estimate the rotation and translation parameters relating two transmitter maps in Channel-SLAM. Within this paper, we extend this approach by using a variant of random sample consensus (RANSAC) [7] in order to increase the robustness and accuracy of map matching. RANSAC is an algorithm widely used in computer vision for image registration, for example. Its fundamental goal is to find the parameters of a mathematical model given a data set with outliers. In our respect, the data set are the transmitter locations, and the model parameters are the rotation and translation relating two coordinate systems. There are numerous variations and extensions of the original RANSAC algorithm in the literature [8], [9].

The remainder of the paper is organized as follows. Section $\Pi$ introduces the basic ideas of multipath assisted positioning and Channel-SLAM. In Section III, we explain RANSAC and derive its adaption to the map matching problem. Evaluations based on simulations in an indoor scenario are presented 


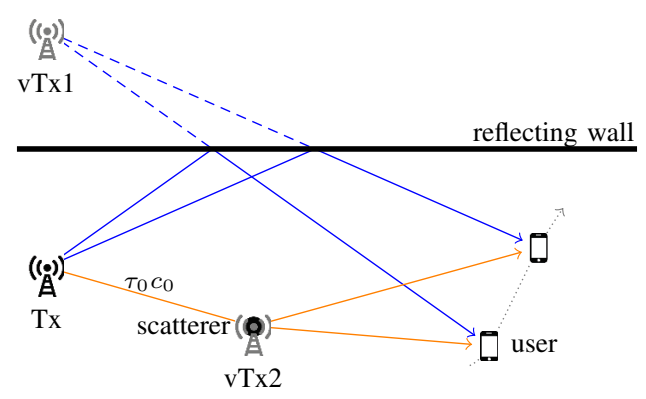

Fig. 1. The signal from the physical transmitter Tx is received by the user via two propagation paths. The signal component reflected at the wall can be regarded as a LoS signal from the virtual transmitter vTx1, and the signal component scattered at the point scatterer as a LoS signal from the virtual transmitter vTx2. In the latter case, there is a time offset $\tau_{0}$ between the physical transmitter and the virtual transmitter vTx2. It is the Euclidean distance between the two transmitters divided by the speed of light $c_{0}$.

in Section IV Section $\mathrm{V}$ concludes the paper.

\section{Multipath Assisted Positioning}

\section{A. Virtual Transmitters}

Fig. 1 illustrates the idea of virtual transmitters. The physical transmitter Tx transmits a signal. This signal is reflected at the wall and received by the user as a MPC. However, this signal can be interpreted by the user as a signal in a pure LoS condition from the virtual transmitter vTx1. This virtual transmitter is located at the location of the physical transmitter mirrored at the wall. The location of vTx 1 is therefore static and independent from the position of the user. Since the propagation distances of a signal from the physical transmitter to the user and from the virtual transmitter to the user are exactly the same, the two transmitters are inherently time synchronized.

At the same time, the signal from the physical transmitter is scattered at a point scatterer and received by the user as a MPC. Again, this received signal is regarded as a signal in a LoS condition from the virtual transmitter vTx2. In the case of scattering, the location of the virtual transmitter coincides with the point scatterer's location. The location of the virtual transmitter is therefore again static. However, the virtual transmitter has a time delay $\tau_{0}$ towards the physical transmitter. This time delay is the Euclidean distance between the physical and the virtual transmitter divided by the speed of light $c_{0}$. The time delay can be interpreted as a clock offset between the two transmitters.

It is straightforward to extend this model of virtual transmitters to the case where a signal is reflected and/or scattered multiple times [5].

\section{B. Channel-SLAM}

We model the propagation channel between the physical transmitter and a mobile user as a linear multipath channel. The transmit signal arrives at the user via different propagation paths as a superposition of different signal components. Each signal component has a delay $d_{i}(t)$ and a complex amplitude $\alpha_{i}(t)$ at time $t$. The channel impulse response (CIR) $h(\tau, t)$ at time $t$ with delay $\tau$ is therefore

$$
h(\tau, t)=\sum_{i} \alpha_{i}(t) \delta\left(\tau-d_{i}(t)\right),
$$

where $\delta(\cdot)$ denotes the Dirac delta distribution.

Channel-SLAM works in two stages. In the first stage, after sampling the received signal, a channel estimator estimates the parameters of the signal components. Within the scope of this paper, we use the time of arrival (ToA) and angle of arrival (AoA) estimates assuming an antenna array at the receiver. We use the channel estimator named Kalman enhanced super resolution tracking (KEST) [10]. KEST uses snapshotbased signal parameter estimates obtained from the SpaceAlternating Generalized Expectation-Maximization (SAGE) [11] algorithm and tracks these parameters over time with Kalman filters [12]. In addition, it keeps track of the number of detected signal components $N_{\mathrm{TX}}$ at the receiver. At time instant $k$, the ToA estimates

$$
\boldsymbol{d}_{k}=\left[\begin{array}{lll}
d_{1, k} & \ldots & d_{N_{\mathrm{TX}}, k}
\end{array}\right]^{T}
$$

and the AoA estimates

$$
\boldsymbol{\theta}_{k}=\left[\begin{array}{lll}
\theta_{1, k} & \ldots & \theta_{N_{\mathrm{TX}}, k}
\end{array}\right]^{T}
$$

are stacked in the measurement vector

$$
\boldsymbol{z}_{k}=\left[\begin{array}{ll}
\boldsymbol{d}_{k}^{T} & \boldsymbol{\theta}_{k}^{T}
\end{array}\right]^{T} .
$$

In the second stage of Channel-SLAM, the estimates from KEST in the first stage are used to localize the user and estimate the states of the physical and virtual transmitters with SLAM. Channel-SLAM does not differentiate between physical and virtual transmitters. Each arriving signal component, no matter if LoS component or MPC, corresponds to one transmitter. Thus, there is no differentiation between physical and virtual transmitters, and the term transmitter will generally denote either of them in the following.

Since physical and virtual transmitters are static in our model as in Subsection II-A each transmitter is described by its location in two dimensions and a clock offset. Hence, we have for the state vector of the $j^{\text {th }}$ transmitter at time instant $k$

$$
\boldsymbol{x}_{\mathrm{TX}, k}^{<j>}=\left[\begin{array}{lll}
x_{\mathrm{TX}, k}^{<j>} & y_{\mathrm{TX}, k}^{<j>} & \tau_{0, k}^{<j>}
\end{array}\right]^{T},
$$

where $x_{\mathrm{TX}, k}^{<j>}$ and $y_{\mathrm{TX}, k}^{<j>}$ specify the location and $\tau_{0, k}^{<j>}$ the clock offset of the transmitter. The user state comprises the location and velocity in two dimensions, i.e., the user state vector at time instant $k$ is denoted by

$$
\boldsymbol{x}_{\mathrm{u}, k}=\left[\begin{array}{llll}
x_{k} & y_{k} & v_{x, k} & v_{y, k}
\end{array}\right]^{T} .
$$

Finally, the state vector $\boldsymbol{x}_{k}$ at time instant $k$ consists of the user state $\boldsymbol{x}_{\mathrm{u}, k}$ and the transmitters' state $\boldsymbol{x}_{\mathrm{TX}, k}$, i.e.,

$$
\begin{aligned}
\boldsymbol{x}_{k} & =\left[\begin{array}{llll}
\boldsymbol{x}_{\mathrm{u}, k}^{T} & \boldsymbol{x}_{\mathrm{TX}, k}^{T}
\end{array}\right]^{T} \\
& =\left[\begin{array}{llll}
\boldsymbol{x}_{\mathrm{u}, k}^{T} & \boldsymbol{x}_{\mathrm{TX}, k}^{<1>T} & \ldots & \boldsymbol{x}_{\mathrm{TX}, k}^{<N_{\mathrm{TX}}>T}
\end{array}\right]^{T} .
\end{aligned}
$$


Note that the number $N_{\text {TX }}$ of signal components, or transmitters, may change over time. For notational brevity, though, we omit the time index $k$ in $N_{\mathrm{TX}}$.

We seek to estimate the posterior distribution $\mathrm{p}\left(\boldsymbol{x}_{0: k} \mid \boldsymbol{z}_{1: k}, \boldsymbol{u}_{1: k}\right)$, with $\boldsymbol{x}_{0: k}$ denoting the state vector from time instants zero to $k$ and $z_{1: k}$ denoting the measurements from time instants one to $k$. The control input $\boldsymbol{u}_{1: k}$ can be obtained from an inertial measurement unit (IMU) carried by the user, for example. The posterior distribution can be factorized as

$$
\begin{aligned}
\mathrm{p}\left(\boldsymbol{x}_{0: k} \mid \boldsymbol{z}_{1: k}, \boldsymbol{u}_{1: k}\right)= & \mathrm{p}\left(\boldsymbol{x}_{\mathrm{TX}, 0: k}, \boldsymbol{x}_{\mathrm{u}, 0: k} \mid \boldsymbol{z}_{1: k}, \boldsymbol{u}_{1: k}\right) \\
= & \mathrm{p}\left(\boldsymbol{x}_{\mathrm{u}, 0: k} \mid \boldsymbol{z}_{1: k}, \boldsymbol{u}_{1: k}\right) \\
& \times \mathrm{p}\left(\boldsymbol{x}_{\mathrm{TX}, 0: k} \mid \boldsymbol{z}_{1: k}, \boldsymbol{x}_{\mathrm{u}, 0: k}\right) \\
= & \mathrm{p}\left(\boldsymbol{x}_{\mathrm{u}, 0: k} \mid \boldsymbol{z}_{1: k}, \boldsymbol{u}_{1: k}\right) \\
& \times \prod_{j=1}^{N_{\mathrm{TX}}} \mathrm{p}\left(\boldsymbol{x}_{\mathrm{TX}, 0: k}^{<j>} \mid \boldsymbol{x}_{\mathrm{u}, 0: k}, \boldsymbol{z}_{1: k}\right),
\end{aligned}
$$

assuming that the measurements for single transmitters, i.e., the estimates from the channel estimator for different signal components, are independent from each other. The first factor in the last line of Eq. (8) is the posterior of the user state, and the second factor is the product of all transmitter posteriors conditioned on the state of the user. The assumption of independent measurements allows for estimating the state of each transmitter independently from the other transmitters' states.

We apply Bayesian recursive estimation [12] to obtain the posterior distribution. In the prediction step of the user, different movement models and additional sensor data, for example from an IMU, can be incorporated as measurements or control input $\boldsymbol{u}$. Since the transmitters are static in our model, the transition prior for the transmitters is expressed with the identity function. In the update step, we assume white Gaussian noise (WGN) in the ToA estimates and the noise in the AoA estimates following a von Mises distribution.

To cope with the non-linear measurement model and nonGaussian noise, we use a Rao-Blackwellized particle filter [13] to estimate the state vector $\boldsymbol{x}_{k}$. The user state vector $\boldsymbol{x}_{\mathrm{u}, k}$ with posterior distribution $\mathrm{p}\left(\boldsymbol{x}_{\mathrm{u}, 0: k} \mid \boldsymbol{z}_{1: k}\right)$ is estimated by a particle filter, which we call user particle filter. For each user particle, the transmitters' state vector $\boldsymbol{x}_{\mathrm{TX}, k}$ is estimated independently from the other user particles. Assuming independence among measurements for different transmitters, each of the user particles estimates the state of each transmitter independently from the other transmitters by a particle filter.

Thus, the user posterior distribution $\mathrm{p}\left(\boldsymbol{x}_{\mathrm{u}, k} \mid \boldsymbol{z}_{1: k}\right)$ is represented by a sum of $N_{p}$ particles, where the $i^{\text {th }}$ particle $\boldsymbol{x}_{\mathrm{u}, k}^{<i>}$ has a weight $w_{k}^{<i>}$ associated to it,

$$
\mathrm{p}\left(\boldsymbol{x}_{\mathrm{u}, k} \mid \boldsymbol{z}_{1: k}, \boldsymbol{u}_{1: k}\right)=\sum_{i=1}^{N_{p}} w_{k}^{<i>} \delta\left(\boldsymbol{x}_{\mathrm{u}, k}-\boldsymbol{x}_{\mathrm{u}, k}^{<i>}\right) .
$$

Likewise, the posterior distribution, or state probability density function (PDF), of the $j^{\text {th }}$ transmitter of the $i^{\text {th }}$ user

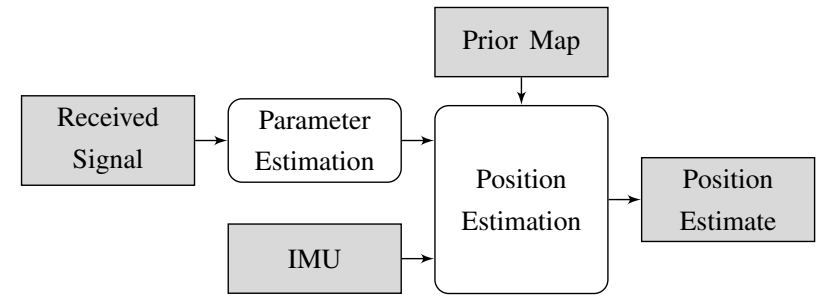

Fig. 2. Overview of the Channel-SLAM algorithm. The parameters of the signal components from the channel estimator, a prior transmitter map and data from additional sensors, such as an IMU, are fused in the particle filter performing the position estimation.

particle is represented by a sum of $N_{p, \text { Tx }}$ particles, where the $l^{\text {th }}$ particle $\boldsymbol{x}_{\mathrm{TX}, k}^{<i, j>}$ has a weight $w_{k}^{<i, j, l>}$ associated to it,

$\mathrm{p}\left(\boldsymbol{x}_{\mathrm{TX}, k}^{<i, j>} \mid \boldsymbol{x}_{\mathrm{u}, k}^{<i>}, \boldsymbol{z}_{1: k}\right)=\sum_{l=1}^{N_{p, \mathrm{Tx}}} w_{k}^{<i, j, l>} \delta\left(\boldsymbol{x}_{\mathrm{TX}, k}^{<i, j>}-\boldsymbol{x}_{\mathrm{TX}, k}^{<i, j, l>}\right)$.

The number of transmitter particles may vary for different transmitters, user particles and time instants. Nevertheless, we omit the corresponding indizes in $N_{p \text {,Tx }}$ for notational brevity. A full derivation of the Channel-SLAM algorithm including the derivation of the weight updates can be found in [5].

Fig. 2 summarizes the two stages of Channel-SLAM. Based on the received signal, the channel estimator estimates the parameters of signal components and tracks them over time. These estimates are fused in the position estimation step with additional sensor data in a particle filter.

\section{MAP MATCHING WITH RANSAC}

In certain scenarios such as in malls, museums, or public buildings, multiple users travel through the same scenario on different trajectories. As each user creates a map in ChannelSLAM, these maps can be shared among users to increase the positioning performance and decrease the convergence time in SLAM. However, Channel-SLAM is a relative positioning approach in the sense that each user creates their own local coordinate system. When transmitter maps are exchanged, their coordinate systems are related by an unknown rotation parameter $\beta$ and unknown translation parameters $\bar{x}$ and $\bar{y}$. In addition, it is in general unknown which transmitters in one map correspond to which transmitters in the other map. We refer to estimating the rotation and translation parameters and the set of corresponding transmitters in two maps as map matching.

In the following, we call a map of transmitters with states estimated by the user a user map. A set of transmitter state estimates that the user has obtained from a different user as prior information is denoted by the term prior map.

\section{A. Finding a Map Match}

In [6], we have proposed a first scheme to perform map matching between a user map and a prior map, which will be briefly reviewed in the following.

In a first step, the correspondences among transmitters in the two map must be found. While a user has no information 
on the absolute locations of the transmitters in the prior map, the information on the relative locations of the transmitters within each map can be exploited. In particular, the relative distances among transmitters within each map are regarded. Based on these relative distances, the correspondences among transmitters are found by a least squares solution.

The shape of a transmitter state PDF depends on the user trajectory and the corresponding geometrical delusion of precision (GDoP). Thus, the shapes of the state PDFs of one transmitter estimated by two different users may differ considerably, and a divergence such as the Kullback-Leibler divergence between the two transmitter estimates may be misleading. Thus, we use only transmitters whose variances of the state PDFs are below a threshold $\delta_{\sigma}$ for map matching, and the distance between two transmitters is defined as the Euclidean distance between the means of their state PDFs.

Let $\boldsymbol{\mu}_{j}^{u}=\left[\begin{array}{lll}x_{j}^{u} & y_{j}^{u} & \tau_{j}^{u}\end{array}\right]^{T}$ be the mean of the state PDF of the $j^{\text {th }}$ transmitter in the user map. Likewise, $\boldsymbol{\mu}_{j}^{p}=\left[\begin{array}{lll}x_{j}^{p} & y_{j}^{p} & \tau_{j}^{p}\end{array}\right]^{T}$ denotes the mean of the corresponding transmitter's state PDF in the prior map. We stack up the means of $N_{T}$ transmitters' state PDFs from the user map in the matrix

$$
\boldsymbol{M}_{\mathrm{u}}=\left[\begin{array}{lll}
\boldsymbol{\mu}_{1}^{u} & \cdots & \boldsymbol{\mu}_{N_{T}}^{u}
\end{array}\right]^{T}
$$

and accordingly the means of the $N_{T}$ transmitters' state PDFs from the prior map in

$$
\boldsymbol{M}_{\mathrm{p}}=\left[\begin{array}{lll}
\boldsymbol{\mu}_{1}^{p} & \ldots & \boldsymbol{\mu}_{N_{T}}^{p}
\end{array}\right]^{T} .
$$

The rotation matrix $\boldsymbol{R}_{\boldsymbol{\beta}}$ is defined as

$$
\boldsymbol{R}_{\boldsymbol{\beta}}=\left[\begin{array}{ccc}
\cos \beta & \sin \beta & 0 \\
-\sin \beta & \cos \beta & 0 \\
0 & 0 & 1
\end{array}\right]
$$

With $\gamma=\left[\begin{array}{lll}\bar{x} & \bar{y} & 0\end{array}\right]$ and $\boldsymbol{J}=\left[\begin{array}{lll}1 & \ldots & 1\end{array}\right]^{T}$, which is of dimensions $N_{T} \times 1$, the relation between the user map and the prior map can be expressed as

$$
M_{\mathrm{u}}=\boldsymbol{M}_{\mathrm{p}} \boldsymbol{R}_{\boldsymbol{\beta}}+\boldsymbol{J} \boldsymbol{\gamma}+\boldsymbol{E},
$$

where $\boldsymbol{E}$ is a residual matrix. The unknown rotation parameter $\beta$ and the translation parameters $\bar{x}$ and $\bar{y}$ can be obtained by minimizing the match error, which is the trace of the matrix $\boldsymbol{E}^{T} \boldsymbol{C}^{-1} \boldsymbol{E}$, in a least square sense. The matrix $\boldsymbol{C}$ is a diagonal matrix containing the one-dimensional variances of the corresponding transmitters. With $C$, the transmitters are weighted for map matching: the reliability of transmitters with small variance is higher than the reliability of transmitters with a high variance.

The above method for finding a map match requires the number $N_{T}$ of transmitters in $\boldsymbol{M}_{\mathrm{u}}$ and $\boldsymbol{M}_{\mathrm{p}}$ to be the same, and that for each transmitter in the user map, there is a correspondence in the prior map. Both conditions are usually not met in practice, as some transmitters observed by one user may not be observed by another user and vice versa, depending on the corresponding user trajectories. Thus, in [6], map matching is performed for all subsets of $N_{T}$ transmitters in the user map and in the prior map, resulting in a huge computational complexity if many transmitters have been observed. The parameters for which the match error $\operatorname{Tr}\left(\boldsymbol{E}^{T} \boldsymbol{C}^{-1} \boldsymbol{E}\right)$ is the smallest are chosen.

\section{B. RANSAC in Channel-SLAM}

We propose to use a variant of the RANSAC [7] algorithm for map matching. The fundamental goal of RANSAC is to estimate the parameters of a mathematical model based on a set of data points corrupted by outliers. Outliers are data points that do not fit the model. The generic algorithm works as follows. Subsets of the data set are randomly chosen assuming that these data contain only inliers, i.e., data being consistent with the underlying mathematical model. Based on the subsets, the parameters for the mathematical model are estimated and evaluated with the entire data set. The set of data points that fit the model is defined as the consensus set. The above procedure is repeated multiple times, and the model parameters calculated with the in some sense best consensus set are returned. The algorithm is summarized in Algorithm 1

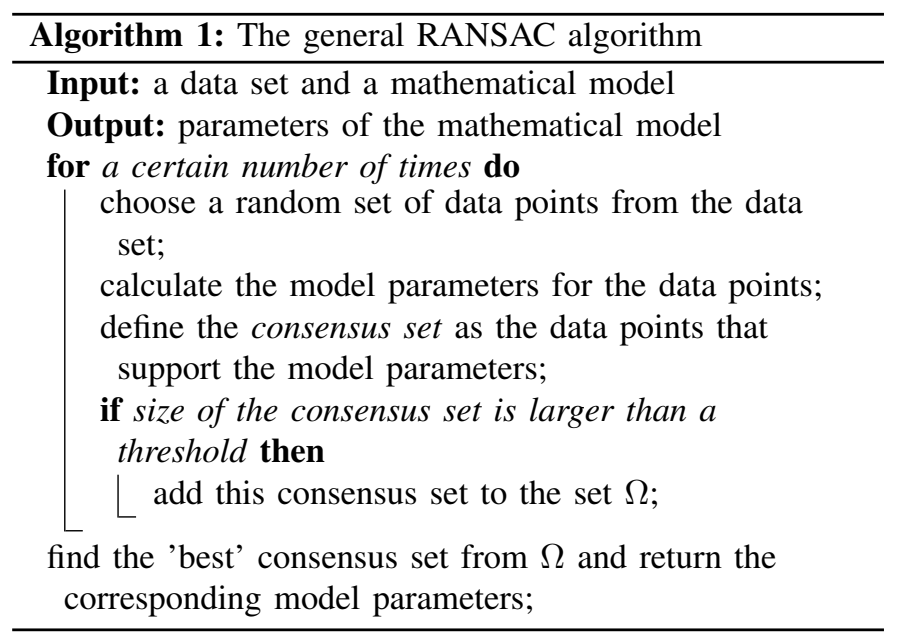

The ability to determine inliers and outliers makes RANSAC a very powerful tool for map matching. For map matching, inliers are transmitters that can be found in both the user and the prior map, whereas outliers are transmitters that are in one of the two maps only. The mathematical model is given by Eq. (14), and the parameters of interest are the rotation $\beta$ and translation $\gamma$. The numbers of transmitters in the user and in the prior map are denoted by $N_{\mathrm{U}}$ and $N_{\mathrm{P}}$, respectively. The data set is the set of means of the transmitter state PDFs in the user and the prior map. The set

$$
\mathrm{D}=\{\mathrm{U}, \mathrm{P}\}
$$

is the combination of the set $U$ of transmitter indices from the user map and the set $\mathrm{P}$ of transmitter indices from the prior map, i.e.,

$$
\begin{aligned}
& \mathrm{U}=\left\{\mathrm{u}_{1}, \ldots, \mathrm{u}_{N_{\mathrm{U}}}\right\} \\
& \mathrm{P}=\left\{\mathrm{p}_{1}, \ldots, \mathrm{p}_{N_{\mathrm{P}}}\right\} .
\end{aligned}
$$




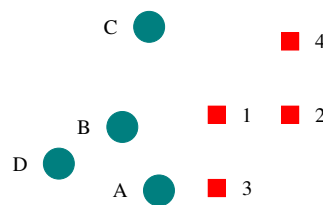

(a)

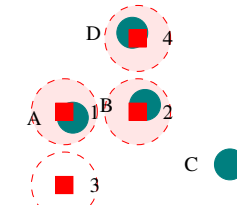

(b)

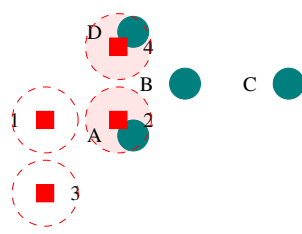

(c)

Fig. 3. In (a), the means of transmitters 1 to 4 from the user map and the means of transmitters A to D from the prior map are drawn in red rectangles and green circles, respectively. A map match with $N_{C}=3$ is shown in (b), a map match with $N_{C}=2$ is shown in (c). The dashed red circles represent the threshold $\delta_{d}$. Filled red circles indicate transmitters from the user and prior map in the consensus set.

A consensus set $\mathrm{C}$ of cardinality $N_{\mathrm{C}}$ is described by the set of tuples

$$
\mathrm{C}=\left\{\left(\mathrm{q}_{1}, \mathrm{r}_{1}\right), \ldots,\left(\mathrm{q}_{N_{\mathrm{C}}}, \mathrm{r}_{N_{\mathrm{C}}}\right)\right\},
$$

where $\mathrm{q}_{\ell} \in \mathrm{U}$ and $\mathrm{r}_{\ell} \in \mathrm{P}$ as well as $\mathrm{q}_{\ell} \neq \mathrm{q}_{u}$ and $\mathrm{r}_{\ell} \neq \mathrm{r}_{u}$ for $\ell \neq u$. Each tuple in the consensus set describes an association between a transmitter from the user map and a transmitter from the prior map.

An inlier is found if the distance between a transmitter in the user map and a transmitter in the prior map is smaller than a threshold $\delta_{d}$ after the rotation and translation. Thus, the tuple $\left(q_{\ell}, r_{\ell}\right)$ is added to the consensus set if

$$
\left\|\boldsymbol{\mu}_{\mathbf{q}_{\ell}}^{u}-\tilde{\boldsymbol{\mu}}_{\mathrm{r}_{\ell}}^{p}\right\|<\delta_{d}
$$

where

$$
\tilde{\boldsymbol{\mu}}_{\mathbf{r}_{\ell}}^{p}=\left(\boldsymbol{\mu}_{\mathbf{r}_{\ell}}^{p T} \boldsymbol{R}_{\boldsymbol{\beta}}+\gamma\right)^{T}
$$

is the mean of the $r_{\ell}^{\text {th }}$ transmitter state PDF from the prior map transformed according to the model in Eq. (14) and $\|\cdot\|$ denotes the Euclidean norm.

The idea of RANSAC for map matching is illustrated in Fig. 3. In Fig. 3 (a), the red rectangles represent the transmitters 1 to 4 from the user map, and the green circles the transmitters A to D from the prior map. After map matching, a map match has been found in Fig. 3 (b), where the dashed red circles have the radius $\delta_{d}$. There are $N_{\mathrm{C}}=3$ inliers found, for which the dashed red circles are filled red. The consensus set is thus described by $\mathrm{C}=\{(1, \mathrm{~A}),(2, \mathrm{~B}),(4, \mathrm{D})\}$. Accordingly, in Fig. 3 (c), $\mathrm{C}=\{(2, \mathrm{~A}),(4, \mathrm{D})\}$ with cardinality $N_{\mathrm{C}}=2$.

As mentioned above, RANSAC chooses the best consensus set whose cardinality is above a threshold. Within this paper, we choose the consensus set which minimizes the error

$$
E_{\mathrm{C}}=\frac{1}{N_{\mathrm{C}}} \sum_{\ell=1}^{N_{\mathrm{C}}}\left\|\boldsymbol{\mu}_{\mathrm{q}_{\ell}}^{u}-\tilde{\boldsymbol{\mu}}_{\mathrm{r}_{\ell}}^{p}\right\|-N_{\mathrm{C}} \rho_{\mathrm{rew}},
$$

where $\left(q_{\ell}, r_{\ell}\right) \in C$ and $\rho_{\text {rew }}$ is a reward term. If the error is above a threshold, we consider the solution unreliable and assume that no map match has been found. The reward term decreases the error for consensus sets of high cardinality, since the reliability of a map match tends to be higher the more transmitters are incorporated in the map match.

\section{Local Optimization}

The basic RANSAC algorithm assumes that a model estimated only with inliers is consistent with all other inliers. However, this assumption does not hold in our case, where we have two reasons for noisy or biased data. On the one hand, we regard only the means of the state PDFs of transmitters. Although only transmitters with a variance smaller than the threshold $\delta_{\sigma}$ are used, the means might be biased towards the true transmitter location. On the other hand, bad estimates from the channel estimator KEST may bias the transmitter mean estimates. Thus, a local optimization in RANSAC is applied as in [14].

The optimization is performed in each iteration after the parameters of the mathematical model are estimated. First, all inliers from the entire data set that are consistent with the model are found, i.e., all transmitters that have a correspondence in the other map. Then, the model parameters are reestimated based on these inliers. In [14], further optimization variants are proposed. One variant is to start with a multiple of the threshold $\delta_{d}$ for obtaining the inliers and decrease it iteratively until it reaches $\delta_{d}$. A second variant is an inner RANSAC algorithm sampling only from the inliers, whereas the obtained models are evaluated based on the entire data set. A third variant is the combination of the two above.

The advantages of the local optimization step are twofold. On the one hand, the number of inliers found is increased, which also leads to a faster convergence of RANSAC. In addition, the estimate for the model parameters tends to be more precise.

\section{Using the Prior Map}

When a map match has been found, the information from the prior map can be fused in the particle filter with the measurements $\boldsymbol{z}_{k}$ obtained from KEST as in Fig. 2. Every time a new signal component is detected by KEST, the question arises if this signal component corresponds to a transmitter that has been observed earlier by the user, to a transmitter from the prior map, or to none of the above. In the latter case, a new transmitter needs to be initialized based on the first measurement from KEST. Otherwise, the new transmitter can be initialized directly with the corresponding transmitter in the user or prior map. The above question is referred to as data association. In [15], we have proposed a data association scheme for Channel-SLAM.

\section{Simulations}

To evaluate the methods developed in Section III, we performed simulations in an indoor scenario with a single physical transmitter. A top view on the scenario is depicted 


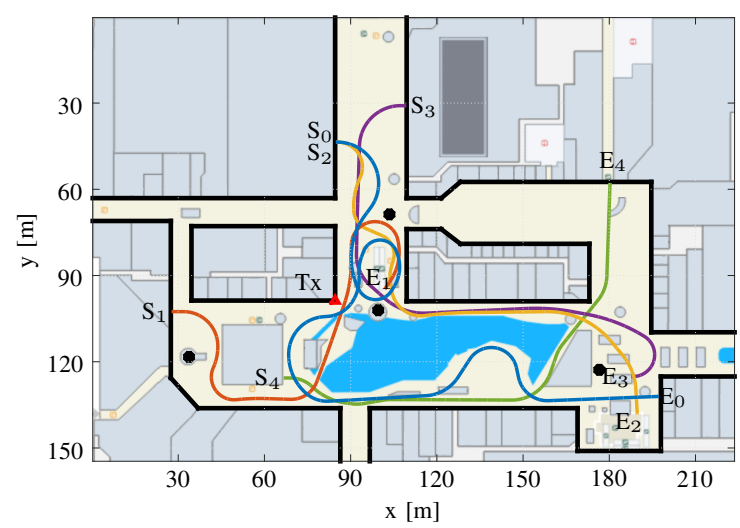

Fig. 4. The indoor simulation scenario with five different user tracks. The $i^{\text {th }}$ user walks along the $i^{\text {th }}$ track starting from $\mathrm{S}_{i}$ finishing at $\mathrm{E}_{i}$. There physical transmitter in the scenario is marked by the red triangle labeled Tx.

in Fig. 4. The physical transmitter location is represented by the red triangle labeled Tx. The transmitter continuously broadcasts a transmit signal of $100 \mathrm{MHz}$ bandwidth. The thick black lines represent walls that reflect the transmit signal. Likewise, the black dots model point scatterers that spread the energy of an impinging signal uniformly to all directions. Within the scope of this paper, we assume that the transmit signal interacts with at most two objects, i.e., we have single and double reflections and/or scattering.

There are five different user tracks in different colors. The $i^{\text {th }}$ user walks along the $i^{\text {th }}$ user track whose start and end points are labeled $S_{i}$ and $E_{i}$, respectively. The users walk with a constant speed of $1 \mathrm{~m} / \mathrm{s}$, and record a snapshot of the received signal every $0.05 \mathrm{~ms}$. The receivers of the users are equipped with two-dimensional antenna arrays consisting of nine elements to obtain both ToA and AoA estimates of the impinging signal components.

The number $N_{p}$ of particles in the user particle filter is 2000. The number $N_{p, \text { Tx }}$ of particles in the transmitter's particle filters is adapted dynamically to the uncertainty of the corresponding transmitter's state with a particle reduction method from [16]. Thus, a transmitter's state PDF with a low uncertainty can be represented with a small number of particles.

For the evaluations, track 0 serves as a reference track. A reference user walks along the reference track with no prior map, i.e., no previous knowledge on the location of any transmitter in the scenario. As the reference user walks through the scenario, they create a map of observed transmitters. This map is handed over to the other four users, i.e. users 1 to 4 . We assume the starting locations of the users to be known, but not their initial heading. Thus, the relative translation between the coordinate systems of the reference user and the other users is known as well, and the relative rotation needs to be estimated. The users 1 to 4 walk along their respective track two times and try to find a map match with the prior map obtained from the reference user. The first time, map matching is performed based on a least squares method as proposed in [6] and described in Subsection [II-A It will be referred to as without RANSAC. The second time, the map matching is performed with the RANSAC algorithm as described in Subsection III-B including the local optimization, and it is referred to as with RANSAC. These two methods are compared against each other in the following.

From the location of the physical transmitter and the scenario in Fig. 4, the locations of the virtual transmitters can be calculated. We calculate the ground truth locations of all virtual transmitters up to an order of two in the scenario, i.e., virtual transmitters that arise due to single and double reflections and/or scattering. For the evaluations, we regard the ground truth transmitter locations for each of the users 1 to 4 rotated by the ground truth angle $\beta$ and the ground truth transmitter locations rotated by the angle $\hat{\beta}$, which was estimated by the corresponding user. In Fig. 5, the distance between any pair of these transmitters is plotted for each user 1 to 4 , i.e., the distance from the transmitter location estimated by the user to the true location. The blue curves show the distances for map matching without RANSAC, and the red curves with RANSAC. The distances can be regarded as an error for the means of the estimated transmitter location state PDFs. We regard only those transmitters that can be observed from the track of the corresponding user. Thus, the overall number of transmitters on the x-axes in Fig. 5 differs from one user to another, and transmitters with the same number from different users might not correspond. The results were averaged over 70 independent simulations for each user.

From Fig. 5 can be observed that the errors with RANSAC are generally significantly smaller than the errors without RANSAC. Thus, the transmitter locations in map matching are estimated with a significantly higher robustness and accuracy with the method proposed in this paper. Note that the absolute values of the errors depend on the locations of the origins of the coordinate systems, since the maps are rotated around the origin. In particular, transmitters of higher orders that arise due to reflections of the transmit signal at distant walls may be spread widely in the scenario. The errors for such transmitters that are far away from the origin of the coordinate system tend to be big even after a rotation by an angle with only a small bias.

\section{CONCLUSION}

In multipath assisted positioning, maps of locations of physical and virtual transmitters can be exchanged among users. The information from such maps can be fused with the observations of the users to increase the positioning performance. Though, since each user is in their own coordinate system, the relative rotation and translation between two maps' coordinate systems have to be estimated based on the transmitters in the maps. Within this paper, we have proposed a variant of RANSAC to estimate this rotation and translation. The ability of RANSAC to deal with outliers, i.e., with transmitters that are in only one of two maps, is very beneficial for our map matching problem as it increases the robustness 

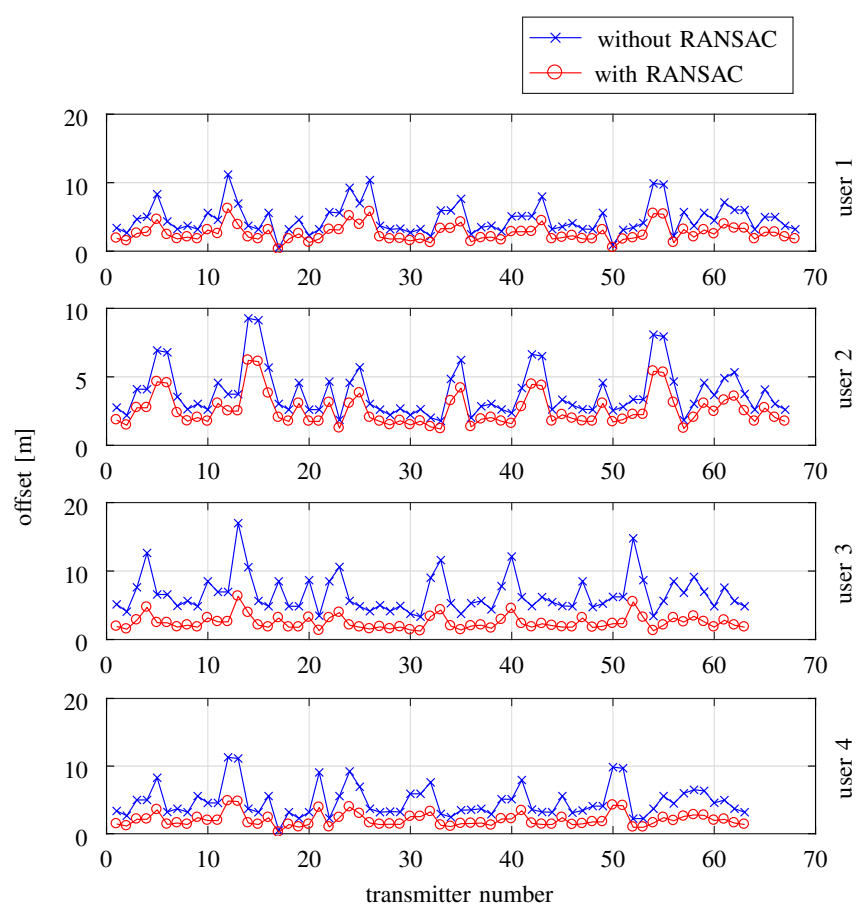

Fig. 5. For each user 1 to 4 , the distances between the true and estimated transmitter locations after map matching are plotted for all transmitters that were observed by the corresponding user. For the blue curves, map matching was performed with a least squares method without RANSAC, and for the red curves with the RANSAC method proposed in the paper.

compared to our previous least squares approach. We showed in simulations that RANSAC increases the accuracy of map matching significantly compared to the standard least squares method.

\section{ACKNOWLEDGEMENT}

This work was partially supported by the the DLR project Navigation 4.0 .

\section{REFERENCES}

[1] F. Gustafsson and F. Gunnarsson, "Mobile positioning using wireless networks: Possibilities and fundamental limitations based on available wireless network measurements," IEEE Signal Process. Mag., vol. 22, no. 4, pp. 41-53, 2005.

[2] K. Yu, I. Sharp, and Y. J. Guo, Ground-Based Wireless Positioning. Wiley-IEEE Press, 2009.

[3] P. Meissner and K. Witrisal, "Multipath-assisted single-anchor indoor localization in an office environment," in Systems, Signals and Image Processing (IWSSIP), 2012 19th International Conference on, Apr. 2012, pp. $22-25$.

[4] P. Setlur, G. Smith, F. Ahmad, and M. Amin, "Target Localization with a Single Sensor via Multipath Exploitation," IEEE Trans. Aerosp. Electron. Syst., vol. 48, no. 3, pp. 1996-2014, Jul. 2012.

[5] C. Gentner, T. Jost, W. Wang, S. Zhang, A. Dammann, and U.-C. Fiebig, "Multipath Assisted Positioning with Simultaneous Localization and Mapping," IEEE Trans. Wireless Commun., vol. 15, no. 9, pp. 6104 6117, Sep. 2016

[6] M. Ulmschneider, D. C. Luz, and C. Gentner, "Exchanging Transmitter Maps in Multipath Assisted Positioning," in IEEE/ION Position, Location and Navigation Symposium (PLANS), 2018.

[7] M. A. Fischler and R. C. Bolles, "Random Sample Consensus: A Paradigm for Model Fitting with Applications To Image Analysis and Automated Cartography," Commun. ACM, vol. 24, no. 6, pp. 381-395, Jun. 1981.

[8] R. Raguram, J.-M. Frahm, and M. Pollefeys, "A comparative analysis of ransac techniques leading to adaptive real-time random sample consensus," in Computer Vision - ECCV 2008, D. Forsyth, P. Torr, and A. Zisserman, Eds. Berlin, Heidelberg: Springer Berlin Heidelberg, 2008, pp. 500-513.

[9] A. Hast, J. Nysjo, and A. Marchetti, "Optimal RANSAC - Towards a Repeatable Algorithm for Finding the Optimal Set," Journal of WSCG, vol. 1, pp. 21-30, 2013.

[10] T. Jost, W. Wang, U. Fiebig, and F. Perez-Fontan, "Detection and Tracking of Mobile Propagation Channel Paths," IEEE Trans. Antennas Propag., vol. 60, no. 10, pp. 4875-4883, Oct. 2012.

[11] B. Fleury, M. Tschudin, R. Heddergott, D. Dahlhaus, and K. Pedersen, "Channel Parameter Estimation in Mobile Radio Environments Using the SAGE Algorithm," IEEE J. Sel. Areas Commun., vol. 17, no. 3, pp. 434-450, Mar. 1999.

[12] S. Kay, Fundamentals of Statistical Signal Processing: Estimation Theory, ser. Fundamentals of Statistical Signal Processing. PrenticeHall PTR, 1998.

[13] A. Doucet, S. Godsill, and C. Andrieu, "On sequential Monte Carlo sampling methods for Bayesian filtering," Statistics and Computing, vol. 10, no. 3, pp. 197-208, Jul. 2000.

[14] O. Chum, J. Matas, and J. Kittler, "Locally Optimized RANSAC," in Pattern Recognition, B. Michaelis and G. Krell, Eds. Berlin, Heidelberg: Springer Berlin Heidelberg, 2003, pp. 236-243.

[15] M. Ulmschneider, C. Gentner, T. Jost, and A. Dammann, "Multiple Hypothesis Data Association for Multipath-Assisted Positioning," in 14th Workshop on Positioning, Navigation and Communications (WPNC), Oct. 2017.

[16] C. Gentner, R. Pöhlmann, M. Ulmschneider, T. Jost, and S. Zhang, "Positioning Using Terrestrial Multipath Signals and Inertial Sensors," Mobile Information Systems, vol. 2017, 2017. 